\title{
Advance Car Alarming System for Fatigue Detection during Driving using MATLAB
}

\author{
Mr. Shreyas Deshmukh ${ }^{1}$, Mr. C.N. Bhoyar ${ }^{2}$ \\ PG Student [VLSI], Dept. of Electronics Engg, PCE, Nagpur, India ${ }^{1}$ \\ Assistant Professor, Dept of Elect \& Tele. Engg, PCE, Nagpur, India ${ }^{2}$
}

\begin{abstract}
The objective of this project is to design an Accident Prevention System which supports in preventing or avoiding accidents. The driver is more disposed to to accidents due to drowsiness and the disturbing intruders. Driver fatigue is one of the supreme common reasons for deadly road accidents around the world. This shows that in the transportation industry especially, where a driver of a heavy vehicle is often exposed to hours of monotonous driving which causes fatigue without frequent rest period. Driver inattention is one of the main causes of traffic accidents. There are lots of approaches and among them computer vision has the potential of monitoring the person behind the wheel without interfering with his driving. The computer vision system for driving monitoring uses face location and tracking as the first processing stage. In the next stage the different facial features are extracted and tracked for monitoring the driver's vigilance. Under this project we will develop a system that can monitor the alertness of drivers in order to prevent people from falling asleep at the wheel System creatively reduces accidents due to drivers' fatigue by focusing on treating the driver later than fatigue has been detect to achieve decline in accident. This paper reviews driver's fatigue monitoring techniques in detail.
\end{abstract}

Keywords: Automatic buzzer system, Distraction detection Drowsiness, fatigue.

\section{INTRODUCTION}

Driver tiredness is a significant feature in a countless number of vehicle accidents. Existing statistics estimate that most of the death and injuries can be accredited to fatigue related crashes. The development of technologies for detecting or to prevent drowsiness at the control is a major challenge in the field of accident escaping systems. Because of the danger that drowsiness present lying on the road, some methods need to be develop for counter acting its affects.

The aspire of this project is to build up a prototype drowsiness detection system. The spotlight will be place on designing a system that will accurately monitor the open or close state of the driver's eyes in real-time. By means of monitoring the eyes, it is thought that the symptom of driver fatigue can be detected early enough to avoid a car accident. Detection of fatigue include a series of images of a face and the observation of eye movement and blink pattern. The analysis of face images is a popular research area with applications such as face recognition, human identification security system and virtual tools. This assignment is focus on the localization of the eyes which include looking at the entire image of the face and defining the position of the eyes by a self-develop image-processing algorithm. Just the once if position of the eyes is located then the system is designed to determine whether the eyes are open or close and detect fatigue.

\section{INFLUENCES RESPONSIBLE FOR DRIVING DROWSINESS}

Tiredness in drivers can be usually separated into the following kinds:

Sensing of driver operation

Sensing of vehicle response.

Monitoring the response of driver.

Sensing of physiological characteristics.

Between these methods, the methods based on human physiological phenomena are the supreme precise.

This technique is implemented in two ways:

Calculating changes in physiological signals, such as brain waves, heart rate, and eye blinking and measuring physical changes such as leaning of the driver's head, sagging posture, and the open/closed states of the eyes.

Drowsiness detection can be divided into four main categories as follows:-

1. Vehicle based measures

2. Behavioral measure

3. Subjective measures

4. Physiological measures 
In the center of the four methods discuss above, the physiological measures type give the precise and consistent product when compare with other methods but it is restricted to implement in real-time. In real-time implementation, vehicle base parameters and behavioral measures have given away decent results in detecting the drowsiness but the dependability in real-time has not been validate.

\section{DETECTION TECHNIQUE FOR DROWSINESS}

Conferring to research, there are manifold categories of technologies which useful to detect driver fatigue. The first is the use of cameras to monitor a person's actions. This includes monitoring mouth for yawning, his pupils, head situation and a variety of additional factors. The next of these technologies is voice detection. Frequently a person's voice can give off clue on how fatigue they are. The detail explanation of the fundamental techniques of drowsiness detection which is mostly use for the detection purpose:

- ECG and EEG

- Local Binary Pattern (LBP)

- Steering Wheel Movement (SWM)

- Optical Detection

\section{FATIGUE DETECTION ALGORITHM}

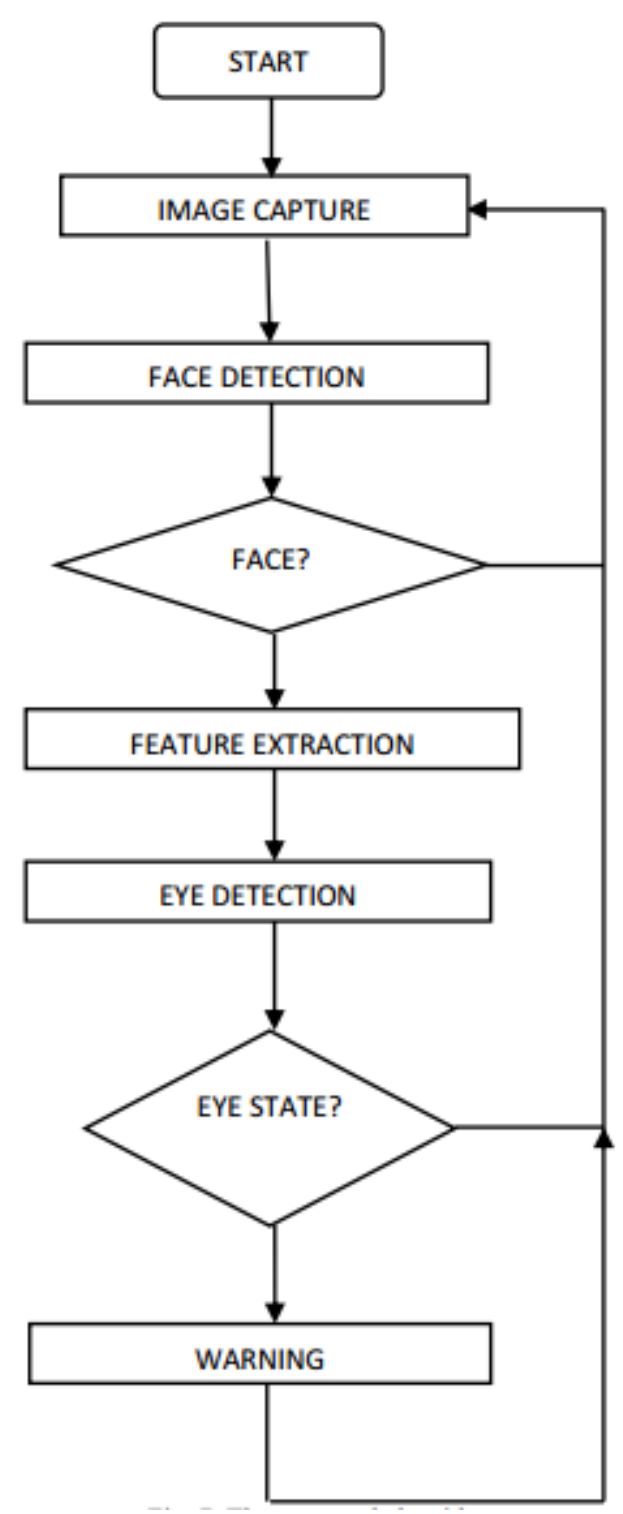

Fig.1 Algorithm for Fatigue Detection 
The major stages of algorithm are as:

1) Image Capture: The image will be captured from the video, where image will be a numeric representation (normally binary) of a two dimensional image. A low cost web camera will acquire the video. The camera will provide 30 frames per second at VGA mode. After that the recorded video will be open in MATLAB and the frames will grabbed, then the algorithm is run on each frame detecting the driver's alertness.

2) Face Detection: In every given frame the face detection will be done using an specified algorithm. Face detection is a computer technology that determines the positions and sizes of human faces in random images. It will detect facial features and will ignore anything else, such as buildings, trees and bodies. Implementation of the facedetection task is done in many algorithms as a binary pattern-classification task. That means, the content of a given element of an image will be transform into features, after which a classifier trained on example faces will decide whether this particular area of the image is a face or not. If the answer of face detection comes optimistic then the algorithm proceed to the next, otherwise the flow of algorithm will go back to the image capture stage.

3) Facial Feature Detection: Facial Feature Detection is to find the accurate location of facial appearance such as eye and mouth corners, jaw contour, lip contour and the shape of the complete face. Face and facial feature detection are difficult problems, due to the large variations a face can have in a sight caused by factors such as intra-subject variations in pose, scale, expression, illumination, background clutter, color, presence of accessories, hair, hats, occlusions, eyeglasses, beard etc. The simple case will consider a single frontal face and will divide it into region of interest like for mouth, nose and eye etc. The feature detection will be used to determine the region of eyes so that their status can be determined easily and quickly. It will be perform by segmenting the face.

4) Eye Detection: Eye detection is the essence of eye tracking and iris recognition. In spite of that there is no other way to identify the eye itself. It seems to be simple but it's really quite complicated. In this process the eyes will be detected in the specified region by the feature detection. In the beginning it will look for the Eigen eye. This process is time taking and it will done just once. After the detection of Eigen eye it will just matched in the other frames for the same candidate.

5) Eyes State: In this stage, it will determine that whether the eyes are closed or open. The detection of eyes status is very important. If the eyes are detected to be close then the warning will sounded. If the eyes are open then the algorithm will goes to the first step of the image capture. The same pattern will repeat to check the status of the eyes.

\section{WORKING BLOCK DIAGRAM}

The block diagram below shows the working principal of the fatigue detection technique.

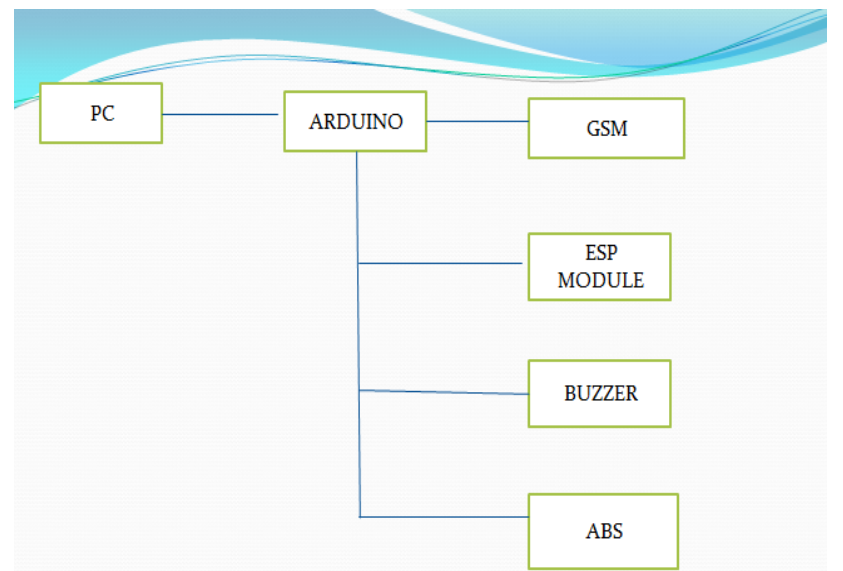

Fig.2 Working Block Diagram of Fatigue Detection Technique

\section{DETECTION OF OTHER COMPONENTS OF THE FACE}

In various driver face nursing systems, other component of driver face such as mouth or nose were detected. However there are a few researches in this field, the methods presented for detection of other components of driver face is reviewed in this section.

1. Mouth Detection:- In certain driver hypo-vigilance detection systems such as, open or closed mouth was used as a measure of driver fatigue. Furthermost of these systems detect the mouth based on red color features of lips. The most significant disadvantage of these methods is that they can only effort properly with color image in visible spectrum and suitable light conditions. 
2. Nose Detection:- Nose tip location with respect to head and eyes is an appropriate criterion for determination of the direction of the head. Three-D model of face can be projected by only one camera and using location of nose tip with respect to eyes. After estimating 3D model of face, determination of head direction is simply possible. Extracted the nostrils for determination of head direction. In this technique, camera was situated slightly lower that driver face while nostrils are often visible. The most important property of nostrils is blackness with respect to face skin. Thus, by applying a threshold on nose area, nostrils are detected. This method is failed for nostrils detection of black people and people who have mustache.

3.Salient Points Detection:- In some recent researches, the salient points of face are detected after face detection. In these researches, the salient points are tracked over time. Thus, according to the relative position of salient points, hypo-vigilance symptoms are extracted. The most common method which is used for salient point detection is ShiTomasi method. This method detects salient angles of a gray-level image as salient points. Tracking and analysis of salient points of face mark the system more robust against occlusion.

4. Tracking of Face and Its Components:- Typically, entire image and entire face region is considered for detection of face and eye respectively. Searching whole image for object detection for all frames of a arrangement increases the computational difficulty of the system. Therefore, usually after detection of the face or eyes at the first frame, face or eye tracking is executed in the next frames. Through assumption of limited movement of face in two consecutive frames, tracking explorations only a limited area of the image instead of the entire image. Thus, tracking usually increase the performance of system. At first, a suitable definition for tracking seems to be necessary. Single definition for tracking was planned as: Tracking is the moving parts of an image in a sequence of frame. Another definition is estimation of motion path of a moving object in successive frames and determination of the object boundary throughout movement.

Two foremost steps in tracking are motion and matching. Motion is estimation of object position in the next frame. Then, matching phase should be performed in the next step. Matching is determination of actual location of the target in the frame based on the estimated location.

\section{EXPERIMENTAL RESULT}

This is an original image of a person with normal activities.

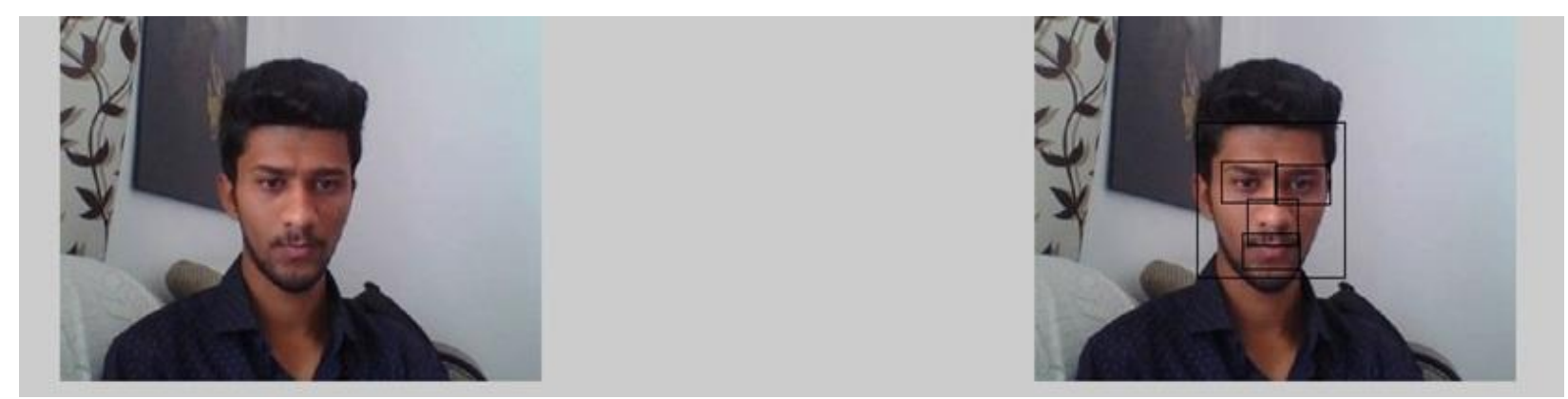

Fig.3 Original image

The figure 4 detects an various face parts like mouth, eyes, nose etc.

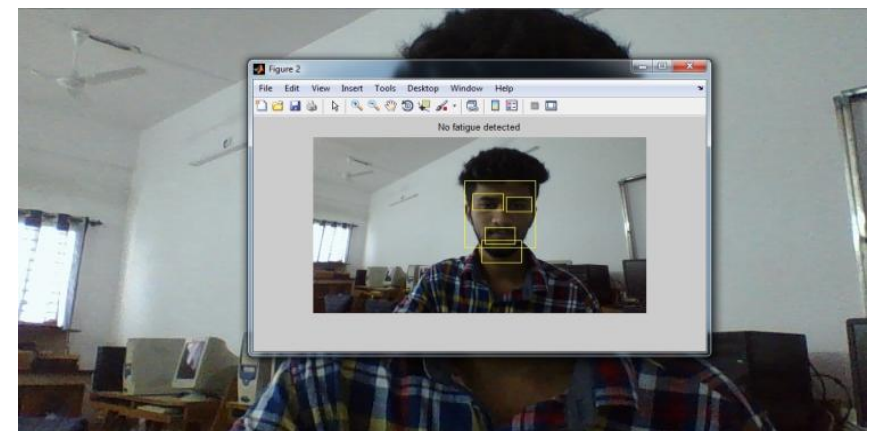

Fig.4 Face Part Locating Image

In this figure, the camera continuesly monitor the face of the driver at steering wheel till it did not find symptoms like fatigue, closure of eyes This image shows that system has correctly detected fatigue face. 


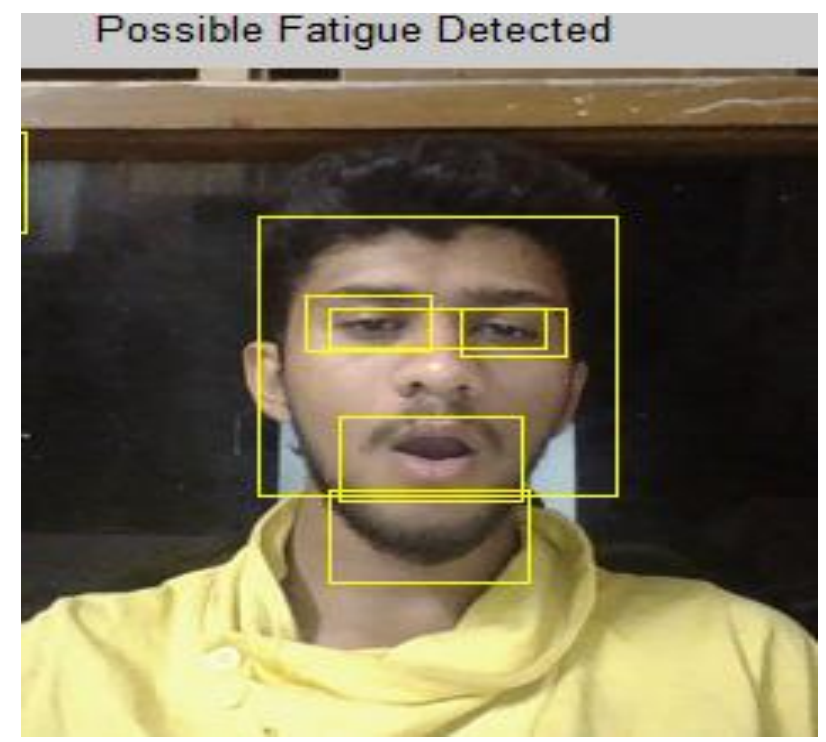

Fig.5 Fatigue Detection Image

Then after the possible fatigue detection, buzzer will beep sound thrice. In case to modification with buzzer we can add vibrator under the drivers seat. And we can send message to driver either on his mobile phone or we can display message on display screen.

\section{CONCLUSION}

The proposed system in this analysis provides accurate detection of driver fatigue. Several technologies be present to distinguish driver fatigue. This paper tries to look at the developing technologies and determine the best methods in trying to prevent the number one cause of fatal vehicle crashes. Driver drowsiness detection systems have been precise advantageous in reducing day by day road accidents. This paper gives a review of driver drowsiness detection technique. The structure is able to recover and properly localize the eyes. Image processing achieves highly accurate and reliable detection of drowsiness. Image processing offers a non-invasive approach to detecting drowsiness.

\section{REFERENCES}

[1] Miss. Monali Gulhane1 and Prof. P. S. Mohod "intelligent fatigue detection and automatic vehicle control system" International Journal of Computer Science \& Information Technology (IJCSIT) Vol 6, No 3, June 2014.

[2] J.Jimenz-pinto and M.Torres-Torriti”Optical Flow And Drivers Kinematics Analysis For State Alert Sensing”,sensors,vol.13,(2013)

[3] Ieee paper on" Driver Fatigue Detection System" by: E. Rogado, J.L. García, R. Barea, L.M. Bergasa, ieee transaction on embedded system vol 54, no.2, may2012.

[4] International journal (IJEAT) paper on "Vision-based Real-time Driver Fatigue Detection System for Efficient Vehicle Control" by D.Jayanthi, M.Bommy ISSN: 2249-8958, Volume-2, Issue-1, October 2012.

[5] Y. Elzohairy, "Fatal and injury fatigue-related crashes on ontario's roads: A 5-year review," Working Together to Understand Driver Fatigue: Report on Symposium Proceedings, February 2008.

[6] T. D’Orazio, M. Leo, C. Guaragnella, and A. Distante, “A visual approach for driver inattention detection,” Pattern Recognition, vol. 40, no. 8, pp. 2341-2355, 2007.

[7] P. Boyraz, M. Acar, and D. Kerr, "Multi-sensor driver drowsiness monitoring," Proceedings of the Institution of Mechanical Engineers, Part D: Journal of Automobile Engineering, vol. 222, no. 11, pp. 2041-2062, 2008.

[8]Y. Matsumoto and A. Zelinsky, "An algorithm for real-time stereo vision implementation of head pose and gaze direction measurements," In Procs. IEEE 4th Int. Conf. Face and Gesture Recognition, pp.499-505, Mar 2000.

[9] A. G. Smart Eye AntiSleep, 2009. [Online]. Available:www.smarteye.se.

[10] W. Shih and Liu, "A calibration-free gaze tracking technique," In Proc. 15th Conf Patterns Recognition, vol. 4, pp. 201-204, Barcelona, Spain, 2000.

[11] Q. Ji and X. J. Yang, "Real-time eye, gaze and face pose tracking for monitoring driver vigilance," Real-Time Imaging, vol. 8, pages 357- 377, Oct 2002.

[12] T. D’Orazio, M. Leo, C. Guaragnella, and A. Distante, “A visual approach for driver inattention detection,” Pattern Recognition, vol. 40, no. 8, pp. 2341-2355, 2007.

[13] P. Boyraz, M. Acar, and D. Kerr, "Multi-sensor driver drowsinessmonitoring," Proceedings of the Institution of Mechanical Engineers, Part D: Journal of Automobile Engineering, vol. 222, no. 11, pp. 2041-2062, 2008. 